\title{
Neighbor-Aided Localization in Vehicular Networks
}

\author{
Susana B. Cruz, Traian E. Abrudan, Member, IEEE, Zhuoling Xiao, Member, IEEE, Niki Trigoni, \\ and João Barros, Senior Member, IEEE
}

\begin{abstract}
We address the problem of localization in vehicular ad hoc networks. Our goal is to leverage vehicle communications and smartphone sensors to improve the overall localization performance. Assuming vehicles are equipped with IEEE 802.11p wireless interfaces, we employ a two-stage Bayesian filter to track the vehicle's position: an unscented Kalman filter for heading estimation using smartphone inertial sensors, and a particle filter that fuses vehicle-to-vehicle signal strength measurements received from mobile anchors whose positions are uncertain, with velocity, GPS position, and map information. Our model leads to a robust localization system and is able to provide useful position information even in the absence of GPS data. We evaluate the algorithm performance using real-world measurements collected from four communicating vehicles in an urban scenario, and considering different combinations of location information sources.
\end{abstract}

Index Terms-Information fusion, road vehicle localization, vehicular ad hoc networks (VANETs), vehicle-to-vehicle (V2V) communications.

\section{INTRODUCTION}

$\mathbf{V}$ EHICULAR ad hoc networks (VANETs) are a promising technology that may provide solutions to many of the current transportation problems, and enable interesting new applications to the users in their everyday lives. Location information plays a key role in several important VANET applications such as geographic information dissemination, traffic control, and automatic positioning of accidents. The focus of this work is on distributed localization of vehicles in a VANET by combining different sources of information. Nowadays, the most widely used positioning service is the Global Positioning System (GPS). However, GPS ceases to work or exhibits large positioning errors in multipath environments and non-line-of-sight conditions to the satellites, such as urban canyons, tunnels, and underground parking [1]. Additional sources of information can be exploited in order to provide better positioning performance for the navigation system. Performance is not exclusively the accuracy, but also integrity, availability, and continuity of service [2].

In VANETs, IEEE $802.11 \mathrm{p}$ communication range typically reaches few hundreds of meters. Deploying a whole network in which any node has a minimum of three road side units (RSU) within reach at all times is highly costly, while lower

Manuscript received (date to be filled in by Editor). This work was supported by UID/EEA/50008/2013, FP7-REGPOT-2012-2013-1, NORTE07-0124-FEDER-000064, and by the Fundação para a Ciência e Tecnologia (Portuguese Foundation for Science and Technology) under grant SFRH/BD/70748/2010.

Susana Cruz and João Barros are with the Instituto de Telecomunicações and Faculdade de Engenharia da Universidade do Porto, 4200-465 Porto, Portugal (e-mails: \{susana.bulas.cruz, jbarros\} @fe.up.pt).

Traian Abrudan, Zhuoling Xiao, and Niki Trigoni are with Department of Computer Science, University of Oxford, OX1 3QD, Oxford, UK (e-mails: \{traian.abrudan, zhuoling.xiao, niki.trigoni\}@cs.ox.ac.uk). density will not provide enough accuracy or coverage [3]. Neighbor-aided localization arises as an alternative approach that overcomes these limitations of localization techniques that rely on fixed anchors alone. Vehicles able to collect information about themselves and about the surrounding environment can act as mobile anchors by sharing information with their neighbors [4]. Our main goal is to leverage communication in VANETs, both between vehicles (V2V) and between vehicles and infrastructure (V2I), and low-cost smartphone sensors in order to improve localization performance. We also use map information, if available, but our algorithm is not dependent on the availability of any of the location information sources. In the absence of GPS, the combination of ranging information from neighbor vehicles and map data is particularly interesting since while the map limits the lateral error, the $\mathrm{V} 2 \mathrm{~V}$ ranging helps to reduce the error along the road direction, where the closest anchors are distributed. We show that this strategy, for a typical urban scenario where the vehicle of interest reaches at least three nearby (maximum distance of $40 \mathrm{~m}$ ) anchors with limited uncertainty ( $\sigma \sigma$ confidence interval of $8 \mathrm{~m}$ ), gives a similar performance to what we obtained using the smartphone's GPS.

The key contributions of this paper are as follows: 1) a new neighbor-aided localization and tracking model using moving vehicles with uncertain positions as anchors that is able to provide reliable position information even in the absence of GPS data; 2) a robust distributed inference algorithm suitable for large-scale use in vehicular networks, fusing various types of data such as V2V signal strength measurements, GPS positions, inertial data from a smartphone, and map information, in a particle filter; and 3) evaluation using real-world data in a challenging urban scenario with quality assessment for different combinations of the location information sources, proposing a characterization for favorable $\mathrm{V} 2 \mathrm{~V}$ ranging conditions. The rest of the paper is organized as follows. Section II provides an overview of the vehicular networks localization literature, focusing mainly on collaborative approaches. We formulate the problem statement in Section III, and the model assumptions in Section IV. Our proposed inference algorithm is explained in Section V, and in Section VI we show experiments with real data to evaluate its performance. Section VII concludes the paper.

\section{OVERVIEW OF LOCALIZATION IN VANETS}

Existing wireless positioning approaches typically rely on pairwise range estimates, and/or relative angles with respect to some anchor nodes whose positions are fixed and known [5]. Range is estimated either from received signal strength indicator (RSSI) measurements using a calibrated channel model [6], 
signal propagation time [7]-[10], or combination of the two [11], whereas angle estimation requires either antenna arrays [5], or coil arrays [12]. Other popular localization techniques are RSSI fingerprinting [13] and simultaneous localization and mapping [14].

Apart from the wireless-based techniques, various on-board sensors may be used to gather useful information for positioning [15]. Vehicle motion sensors such as odometer, velocity encoder, steering encoder, gyroscope, electronic compass, and accelerometer allow the extraction of kinematic information like traveled distance, heading, linear/angular velocity and acceleration. Such sensors are used in inertial navigation systems or dead reckoning techniques [16]. If there is access to digital road maps, the set of possible locations can be reduced and the accuracy of the estimator improved by correcting vehicles' positions and trajectories to the roads, a technique known as map matching. A survey on this topic is provided in [17]. The aim of this paper is to leverage the cheap inertial sensor available in the passenger's mobile phone, as well as vehicleto-vehicle communication, which will most likely become mandatory in future vehicles (e.g. in Europe [18]).

\section{A. Collaborative Localization in VANETs}

Localization in wireless networks is a broad subject. We focus on collaborative approaches [19], [20] for vehicular networks. A comprehensive review on this topic can be found in [21]. The area of robotics also addresses the problem of cooperative localization. However, robots typically share a common goal, and motion and control models differ from the ones for road vehicles. Furthermore, solutions often involve sensors too expensive to be deployed in large scale, such as cameras and lasers [22]. VANETs are a very special type of ad-hoc networks, with particular constraints on vehicle movement, high mobility causing rapid topology changes, and limited bandwidth [23]. These fundamental differences call for specially tailored solutions. Many works address the localization problem in VANETs, pointing out that GPS is insufficient in urban areas. Fusing data from a variety of sources increases not only the accuracy, but also the reliability. In [24], each vehicle shares its own velocity and distance to the neighbors (calculated through RSSI) among its cluster of vehicles. GPS, if available, is used only to define the initial position since the focus is the relative positions of vehicles. Accuracy is increased by using road constraints. Uncertainty along the road is set to be higher than in the orthogonal direction. In [25], every vehicle is equipped with a GPS receiver, an INS and a VANET transceiver. The inter-vehicle communication system extracts information pertinent to the location estimates of vehicles in its vicinity: the distance between the vehicle and its neighbors (GPS based), the location estimates of the neighbors, and their level of uncertainty. Vehicles with the smallest uncertainty are used as anchors, whereas vehicles with detected multipath are not. Both works apply an EKF, which tends to perform better in approximately linear settings (see Section V). Their main limitation is the performance assessment of the proposed model solely on simulations and using a straight highway scenario of few kilometers. These scenarios are very limited as they do not pose most of the challenges faced in real situations, especially in urban settings, such as alternative paths (bifurcations, intersections, roundabouts, etc), sinuous routes, multipath and shadowing effects from numerous obstacles (including moving ones) and non-line of sight communications. By contrast, we use a particle filter, which has the ability to represent arbitrary probability densities, converging to the true posterior asymptotically even in non-Gaussian, nonlinear dynamic systems. This filter is therefore more suitable for our localization approach, evaluated in tortuous urban trajectories and using real measurements.

CoVeL project [26], [27] includes tests with real collected data. The authors use four (valid) vehicles, one of them equipped with a high accuracy GPS and inertial navigation reference system used for ground truth retrieval. $\mathrm{V} 2 \mathrm{~V}$ is solely employed to exchange GPS raw measurements among vehicles to determine their relative positions, assuming that all vehicles experience the same GPS positioning bias and disregarding other errors such as multipath. A group map matching is performed in order to improve accuracy, along with a UKF for position tracking. There are other cooperative localization works that do not use $\mathrm{V} 2 \mathrm{~V}$ ranging techniques, namely [28], [29], focusing instead on improving the lowlevel GPS data. They require at least four pseudo-ranges from visible satellites, making them unsuitable whenever GPS exhibits limited coverage and large positioning error, which happens frequently in dense urban areas. The solution in [30] also uses real data to evaluate performance. However, it makes use of expensive sensors, namely laser scanners, and is therefore beyond the scope of our work, which focuses on approaches with the potential for large-scale application.

\section{PROBLEM Formulation}

Our system consists in a group of vehicles (and RSUs if available), all equipped with IEEE 802.11p vehicular connectivity. Concerning sources of information, we assume that vehicles are heterogeneous and each may have access to different types of data. Examples of potential sources comprise GPS, on-board sensors, road maps, and WLANs signals. In our implementation, we include the first three. The approach is not exclusively developed for urban environments, but focuses on those scenarios since they are more challenging.

We focus on the computations performed in a single vehicle as the center of the network, denoted as $V_{0}$. This vehicle of interest (VOI) aims to calculate its own state. For simplicity, the state of a vehicle consists in its $2 \mathrm{D}$ position and velocity (heading and speed). Our approach can be easily extended to a more complex state, including for example 3D position and acceleration, and to comprise other information sources. Considering the whole network, our goal is to estimate the state of all vehicles, each performing the calculations by seeing itself as the VOI (and its one-hop neighbors as mobile anchors).

We propose a distributed architecture since it is more scalable and flexible for dynamic vehiclular networks than a centralized one. By handling information locally and processing it in the vehicle itself, the computational burden is 
spread among the vehicles. A centralized architecture would imply a high use of resources and be less robust to failure [19] since it would require all measurements to be sent to a central processor that would then transmit the computed results to the vehicles. One limitation of distributed algorithms is the circular reasoning that can arise from inter-estimate dependency, which might lead to over convergence [31]. To avoid this issue, in our work the vehicles do not share any estimation made with external data, i.e. data collected and shared by other vehicles. Each vehicle sends a position estimate obtained only with data acquired from its own sensors, which we name individual position information. This location is sent along with a corresponding measure of uncertainty.

\section{Model Assumptions}

\section{A. Path-loss Channel Model}

In free space, the power of a radio-frequency signal decays proportionally to $d^{2}$, where $d$ is the distance between transmitter and receiver. In real-world channels, however, the propagation of a signal is affected by various phenomena such as reflection, refraction, diffraction, and scattering. It is nonetheless accepted on the basis of empirical evidence that the received signal strength may be modeled as a log-normally distributed random variable with a distance-dependent location parameter [32]. Since the aforementioned effects are environment dependent, the models have to be calibrated for the intended scenario. Eq. (1) provides a path-loss channel model that describes the received signal strength $\rho$ measured in $\mathrm{dBm}$ (decibel milliwatt) given the transmitter-receiver distance $d$ (in meters), using three parameters: received signal strength $\rho_{0}$ $[\mathrm{dBm}]$ at a reference distance of $1 \mathrm{~m}$, channel path-loss exponent $\alpha$, and the fading $v_{\sigma}$, modeled as a zero-mean Gaussian random variable with variance $\sigma^{2}$, i.e., $v_{\sigma} \sim \mathcal{N}\left(0, \sigma^{2}\right)$.

$$
\rho(d)=\rho_{0}-10 \alpha \log _{10}(d)+v_{\sigma}
$$

Fig. 1 shows a set of real measurements along with the estimated path-loss model (using logarithmic scale for the distance, the model becomes linear). The data were collected in an urban scenario, from four vehicles communicating through $802.11 \mathrm{p}$ with $50 \mathrm{~Hz}$ beacons, during a 10 minutes drive of approximately $5.5 \mathrm{~km}$, in Porto, Portugal. The linear model in Fig. 1 was obtained from the measurement data of one of the vehicles using linear regression. The estimated values for the channel parameters are $\rho_{0}=-34 \mathrm{dBm}, \alpha=2.1$, and $\sigma=5.5 \mathrm{~dB}$. Having calculated these channel parameters, we are able to apply the path-loss model to estimate the distances from the RSSI values of the neighbors. Different techniques can be used to estimate the position of the vehicle from the distance to its neighbors. We opted for a parametric approach as it takes into account the uncertainty of the measurements. In order to estimate the location of a vehicle, we calculate the likelihood function $l_{\rho}(d)=p(\rho \mid d)$ corresponding to the path-loss model in (1).

$$
l_{\rho}(d)=\mathcal{N}\left(\rho_{0}-10 \alpha \log _{10}(d), \sigma^{2}\right)
$$

It is important to note that several parameters impact ranging precision, such as the true distance $d$, the fading variance $\sigma^{2}$,

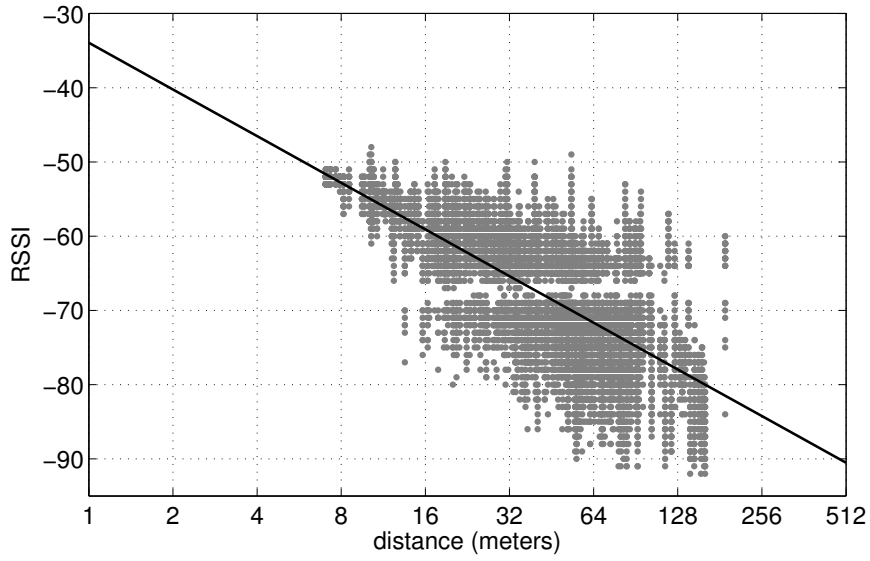

Fig. 1: Pairs of GPS estimated distance and RSSI used to determine channel parameters along with the estimated model.

and the number of available measurements $M$. Below, we provide the Cramér-Rao Lower Bound (CRLB), a lower bound on the variance of any unbiased estimator $\hat{d}$ of $d$ :

$$
\operatorname{CRLB}(\hat{d})=\frac{1}{M}\left(\frac{\sigma d \ln 10}{10 \alpha}\right)^{2} .
$$

Eq. (3) highlights the importance of each parameter in the estimator variance. The CRLB increases quadratically with $d$ and $\sigma$ and is inversely proportional to $M$.

We substitute $d$ by the Euclidean distance between a vehicle position $\mathbf{x}_{0}=\left[x_{0}, y_{0}\right]^{\top}$ and anchor position $\mathbf{x}_{a}=\left[x_{a}, y_{a}\right]^{\top}$ to obtain the likelihood of the location of the VOI $V_{0}$. We assume that $n_{A}$ anchors are available, and that the channel model is the same for all anchors. Since the anchors are at different locations separated by a distance much higher than the wavelength, we also assume that the vehicles experience independent fading. Therefore, the joint likelihood function of the position factorizes as follows:

$$
l_{\rho}\left(\mathbf{x}_{0}\right)=\prod_{a=1}^{n_{A}} \mathcal{N}\left(\rho_{0}-10 \alpha \lg \left\|\mathbf{x}_{a}-\mathbf{x}_{0}\right\|, \sigma^{2}\right) .
$$

The corresponding CRLB for the general scenario with $n_{A}$ anchors, each providing $M_{a}$ independent RSSI measurements, is given by (5). We observe that the number of anchors and their geometry relative to the VOI impact the lowest achievable variance. These results are consistent with the ones presented in [5] for a location estimation algorithm using RSSI, although we show ours from the perspective of a single vehicle, VOI, while in [5] they are calculated for the whole network. In [33], the authors present the CRLB for the scenario of network topology uncertainty. The fundamental limits of wideband localization are presented in [34].

$$
\operatorname{CRLB}\left(\hat{\mathbf{x}}_{0}\right)=\left(\frac{\sigma \ln 10}{10 \alpha}\right)^{2}\left[\sum_{a=1}^{n_{A}} \frac{\left(\mathbf{x}_{a}-\mathbf{x}_{0}\right)\left(\mathbf{x}_{a}-\mathbf{x}_{0}\right)^{\top}}{M_{a}^{-1}\left\|\mathbf{x}_{a}-\mathbf{x}_{0}\right\|^{4}}\right]^{-1}
$$

A higher number of anchors helps to build up the rank of the Fisher information matrix (FIM) by summing up rank-one matrices (outer products) in (5). The FIM rank is increased as long as the corresponding position vectors are linearly 
independent. For example, 2-D position estimation requires a rank-two (invertible) FIM. When the anchors lie approximately on a line, the rank of the FIM tends to one, and therefore the positioning estimator exhibits very large variance along the direction orthogonal to the corresponding line (null space). This conclusion is also consistent with the concept of horizontal dilution of precision (HDOP), which quantifies the impact of range estimation errors on the positioning error given certain anchor configurations.

\section{B. Map Information}

If a road map is available, we distinguish between road and non-road areas. In our implementation we use basic map data from Open Street Map: each road segment is defined simply by two edge points. We consider this segment as the center of the road. The map does not include the width of the roads or their number of lanes. Since our focus is on urban environments, we attribute a default width of $10.5 \mathrm{~m}$ to all road segments, assuming a three-lane road ( $3.5 \mathrm{~m}$ each lane) as the largest possible scenario. Most streets in the city have either one or two lanes (same or opposite directions), so this value already gives us a safe margin by including potential roadsides or parking spaces. By choosing the highest value, we may keep non-road locations flanking narrower roads as valid but we also guarantee that we do not eliminate suitable position candidates. This assumption can be adapted depending on the scenario and the available map details.

\section{INFERENCE ALGORITHM}

\section{A. Bayesian Approach}

Fusing different types of data with different degrees of reliability in a single position estimator can be done in many different ways. Parametric estimation approaches, such as Maximum Likelihood, rely on statistical models associated with erroneous measurements. Based on the assumption that the errors corresponding to different sensors are statically independent, a joint likelihood of all available information can be calculated [35]. Bayesian inference methods can also be applied, involving prior information, and not only the likelihood [36]. Typically, when estimating time-varying parameters filtering algorithms are used, the choice being based upon a trade-off between accuracy and complexity. Kalman Filter (KF) is widely used for its simplicity of implementation, tractability and robustness. When measurement and state transition models are linear and all errors are Gaussian, KF is an optimal estimator in the mean-square sense. However, often the observation model and vehicle dynamics in road navigation are nonlinear processes. Therefore, we focus on nonlinear filtering methods more suitable for navigation. Extended Kalman Filter (EKF) linearizes the nonlinear model with first-order Taylor series about the predicted state, such that KF can be applied. However, the employed models are often highly nonlinear, and divergence may occur, especially when measurements exhibit high noise. Unscented Kalman Filter (UKF) [37] appears as an alternative to EKF, especially for highly nonlinear systems for which the latter gives particularly poor performance. Particle filters, on one hand, impose no restrictions on the state-space model. On the other hand, when the dimension of the state is large, they suffer from "the curse of dimensionality" [38], i.e., large computational complexity. More details on the comparison of nonlinear filters can be found in [39].

Bayesian-filters are powerful statistical tools for state estimation that reliably combine information originating from multiple sources with different degrees of reliability. In order to track the location over time, we employ a two-stage Bayesian filter. The main stage is a particle filter for location tracking. We choose this filter because it allows the representation of arbitrary probability density functions and makes it very easy to incorporate the road restrictions. We are, however, mindful of their potential large complexity and careful to keep the computational cost feasible. Once the posterior function has been calculated, in order to obtain the state estimate, we use a maximum a posteriori estimate, which corresponds to the mode of the posterior density. As our state-space model is highly non-linear, we opt for a bootstrap particle filter that implements a sequential importance sampling with the transition prior probability distribution as importance function [35]. This main filtering stage includes a prediction phase based on the vehicle's dynamics, namely the velocity (speed and heading). A secondary filtering stage, which is a UKF, is employed and outputs the heading estimate required by the main stage. This UKF fuses inertial measurements collected from a smartphone (magnetometer, gyroscope and accelerometer data).

\section{B. Proposed Filter}

In Section IV-A, we considered the method to estimate the position of $V_{0}$ in a fixed time instant, i.e. we did not take the motion of the vehicles into account yet. Now, we focus on the dynamic part of the solution, proposing a two stage inference method. We shall first introduce the statespace model corresponding to the main stage of our Bayesian filter, i.e., the particle filter for position tracking. We define the current full state of the vehicle of interest $V_{0}$ at time $k$ in Cartesian coordinates as

$$
X_{0}(k)=\left[x_{0}(k), y_{0}(k), s_{0}(k), h_{0}(k)\right]^{\top},
$$

where $x_{0}(k), y_{0}(k)$ are the vehicle position coordinates (in meters) at time $k, s_{0}(k)$ is the vehicle speed (in meters/second), and $h_{0}(k)$ denotes the vehicle's heading (measured in radians from the $x$-axis that coincides with the East direction, and positive angles are measured from East to North).

The state-space model of the second stage is given as:

$$
\begin{gathered}
x_{0}(k)=x_{0}(k-1)+T s_{0}(k-1) \cos \left(h_{0}(k-1)\right) \\
y_{0}(k)=y_{0}(k-1)+T s_{0}(k-1) \sin \left(h_{0}(k-1)\right) \\
s_{0}(k)=\hat{s_{0}}(k)+w_{s_{k}} \\
h_{0}(k)=h_{0}(k-1)+\Delta \hat{h}+w_{h_{k}}
\end{gathered}
$$

where $T$ is the time interval between instances $k-1$ and $k$ (in seconds), whereas $\Delta \hat{h}$ is the estimated heading change rate during interval $T$, and $\hat{s_{k}}$ is the estimated average speed within that time interval. The process noise is defined for both heading and speed as Gaussian distributions, represented 
by $w_{h_{k}}$ and $w_{s_{k}}$, respectively. The corresponding normal distributions have zero mean and variances $T \sigma_{h}^{2}$ and $T \sigma_{s}^{2}$, respectively, where $\sigma_{h}$ and $\sigma_{s}$ are the reference standard deviations for an interval of one second.

1) State Equations: The state prediction equation is a simple linear motion model along each coordinate $x$ and $y$, as shown in Eqs. (7) and (8), respectively. We assume the vehicle has access to heading and speed estimates to be used in the motion model. These can be obtained from any source, without loss of generality. For example, the speed information can be collected by a low-cost on-board diagnostics (OBD) device connected to the vehicle. In our case, the heading is estimated using the inertial sensors available in the passenger's smartphone, namely accelerometer, magnetometer and gyroscope. A UKF implementation based on [40] was used, corresponding to the secondary stage of the proposed Bayesian filter. Please refer to Section 5.2 of [40] for details about the algorithm for orientation estimation.

2) Measurement Equations: We define $Z(k)$, a composite measurement vector at time $k$ which is comprised of individual GPS position estimates and RSSI measurements the VOI collected from the available anchors. A different model is necessary for each sensor in composite vector $Z(k)$. For the RSSI, the model is derived from (1), rewriting the distance $d$ in terms of coordinates of the VOI $\mathbf{x}_{0}(k)$ for all available anchors $\mathbf{x}_{a}(k)$. The RSSI measurement $\rho_{a}(k)$ corresponding to the packets sent from an anchor $a$ at time $k$ is related to the vehicle position $\mathbf{x}_{0}(k)$ as follows

$$
\rho_{a}(k)=\rho_{0}-10 \alpha \lg \left\|\mathbf{x}_{a}(k)-\mathbf{x}_{0}(k)\right\|+v_{\sigma}(k),
$$

and the corresponding joint likelihood function for $n_{A}$ anchors is given in (4). In our model, moving vehicles that are in the range of $V_{0}$ act as mobile anchors. They know their positions with some degree of uncertainty and share their own estimated positions $\hat{\mathbf{x}}_{a}(k)=\left[\hat{x}_{a}, \hat{y}_{a}\right]^{\top}$ along with respective uncertainty, e.g. (a representation of) their location posterior density. In the implementation, we used GPS position estimates along with position reliability measure provided by the GPS receiver. Due to anchor position uncertainty, their realizations have been drawn from a Gaussian distribution with mean $\mathbf{x}_{a}$ as the measured GPS position, and standard deviation $\sigma_{a}$ derived from the corresponding position reliability measure. The likelihood corresponding to the anchor's GPS measurements is given by $\mathcal{N}\left(\hat{\mathbf{x}}_{a}(k), \sigma_{a}(k)\right)$, where $\hat{\mathbf{x}}_{a}(k)$ and $\sigma_{a}$ are the estimated GPS position and its standard deviation at time $k$. In the algorithm (see Algorithm 1), in order to reduce the computational cost of calculating the joint likelihood function for $n_{A}$ anchors with uncertain positions online, we precompute it using 100 particles drawn from the aforementioned Gaussian function to represent each anchor and save the results in a lookup table.

If individual location estimates are available at the vehicles, for example from GPS, the corresponding likelihood functions are modeled according to the reliability of these estimates. The composite likelihood function $p(Z(k) \mid X(k))$ is given by

$$
p(Z(k) \mid X(k))=l_{L}\left(\mathbf{x}_{0}(k)\right) l_{\rho}\left(\mathbf{x}_{0}(k)\right)
$$

where $l_{L}\left(\mathbf{x}_{0}(k)\right)$ is the product of the probabilities of $\mathbf{x}_{0}(k)$ being the current position of $\mathrm{V} 0$, for each alternative source

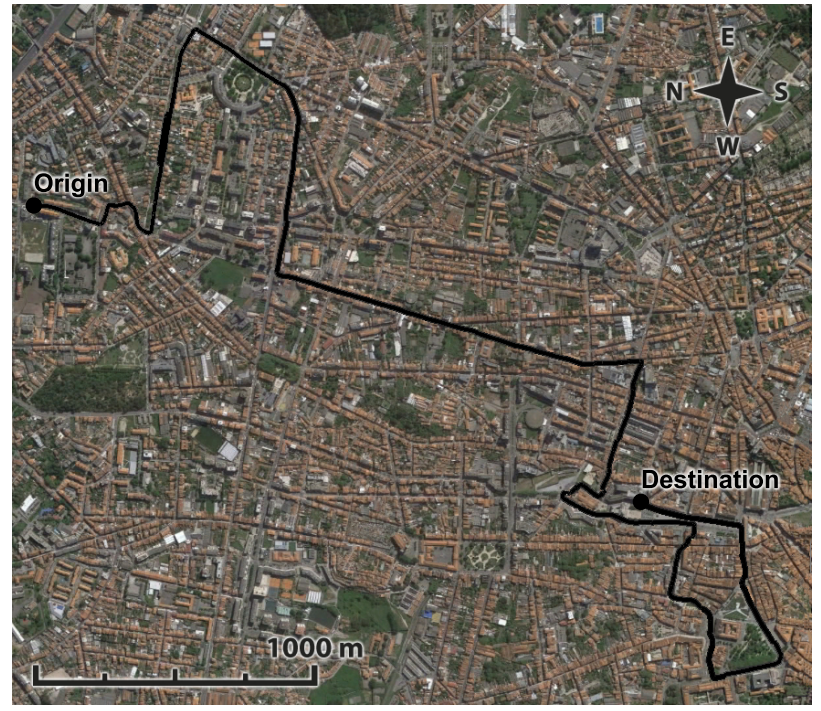

Fig. 2: Urban trajectory of approximately $7 \mathrm{~km}$.

of individual information. The global positions coordinates have been converted to Cartesian ones using an equirectangular projection.

3) Map Restrictions: For each particle, we check whether its position lies on a road or not. Particles outside the roads are deleted and to maintain the total number of particles, the ones on the roads are replicated according to their weights, so that particles with higher weights have higher probability of being replicated.

4) Computational Cost: The computational complexity of our particle filter is $\mathcal{O}\left(n_{A} n_{P}\right)$ at each time instance $k$, where $n_{A}$ is the number of anchors and $n_{P}$ the number of particles. The number of anchors is usually much lower than the number of particles and anchors should be limited to the closest or more promising neighbors (the ones with higher confidence in their position). Therefore the computational cost is mostly driven by the number of particles used in the filter, a tradeoff between the accuracy of the estimation and the required computational resources to achieve it in suitable time. For example, considering an update rate of few $\mathrm{Hz}$, and one thousand particles, the computation can be easily carried out by most modern multi-core processors based smartphones.

\section{EXPERIMENTS WITH REAL DATA}

In this section, we show experiments as a proof-of-concept for our approach in a real world setting and providing an evaluation of quality among different combinations of information sources. Four cars were driven for 30 minutes in the city of Porto along the route shown in Fig. 2, facing everyday traffic conditions with regular driving behavior. No special environments or settings were chosen, other than keeping the vehicles in communication reach of each other for as long as possible while being safe and compliant with the road rules. Each vehicle was equipped with a purposebuilt development platform for vehicle communication, NEC LinkBird MX, which implements $802.11 \mathrm{p}$ wireless standard $(5.85-5.925 \mathrm{GHz})$ and has built-in beaconing functionality of 


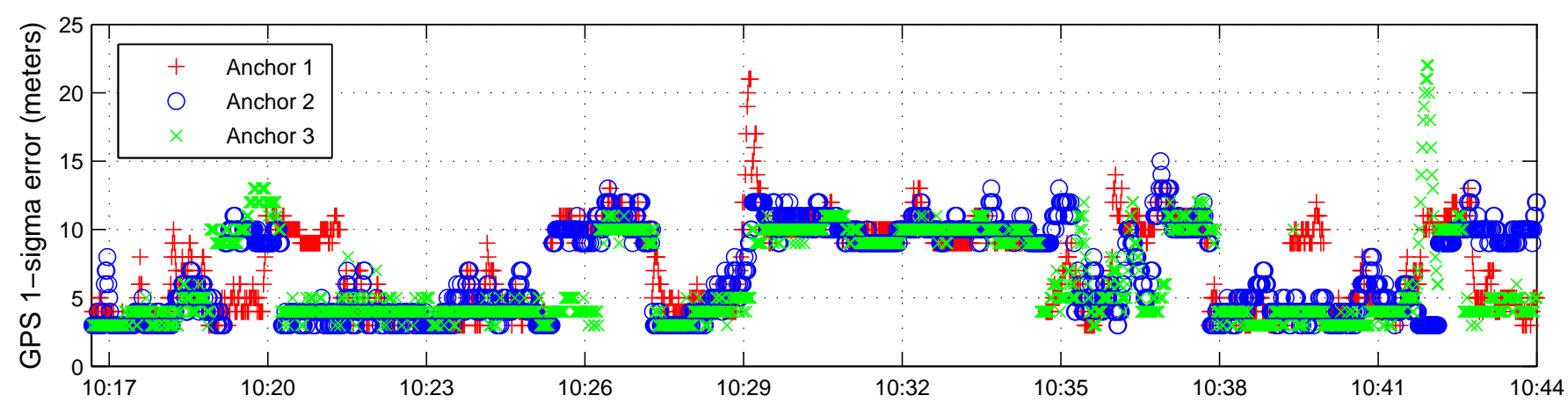

(a) Horizontal $1 \sigma_{a}$ error from each anchor

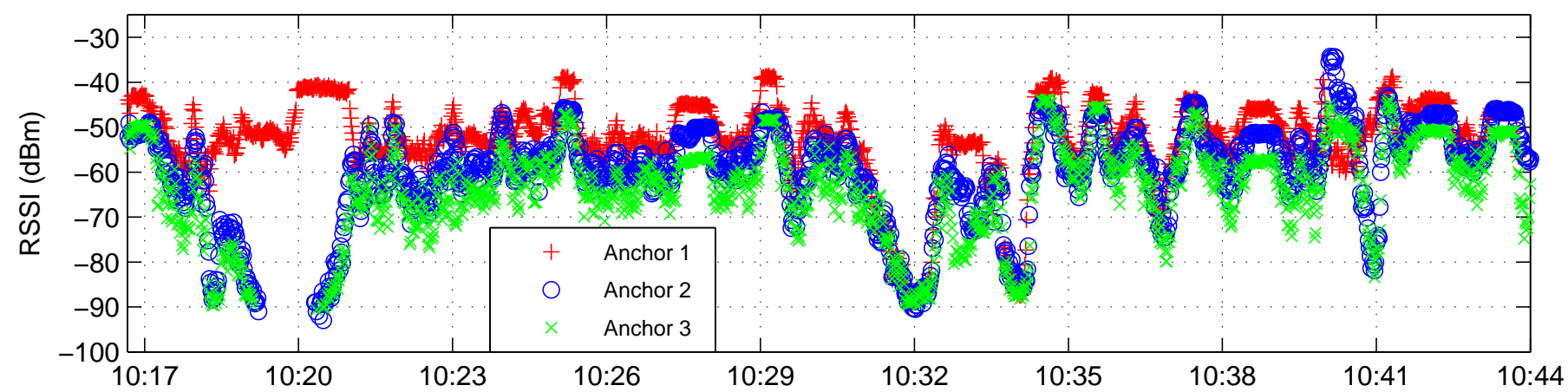

(b) Mean RSSI (using 50 beacons per second) from each anchor

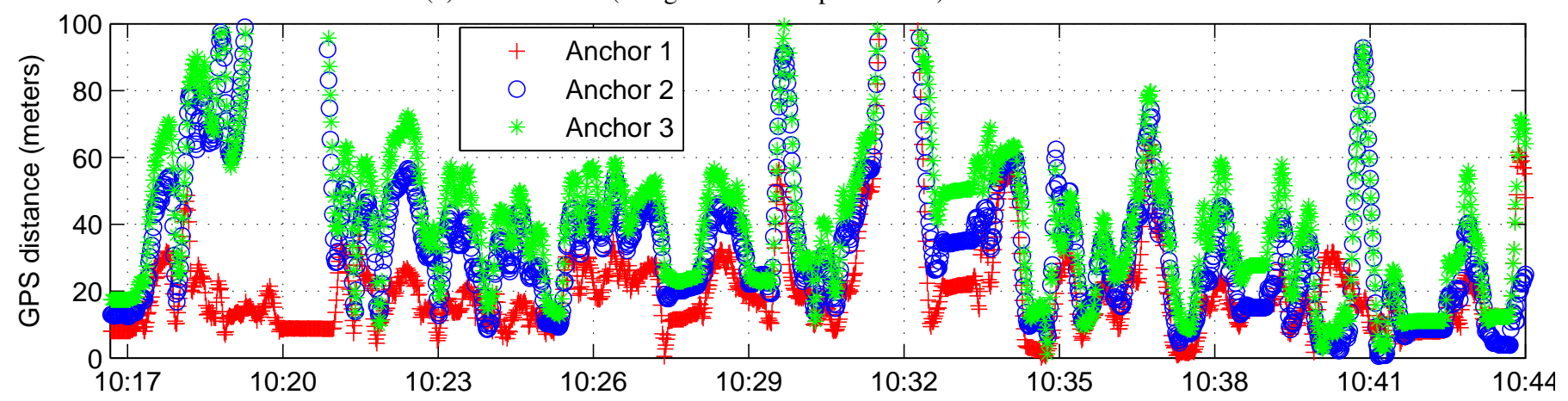

(c) GPS distance from VOI to each anchor

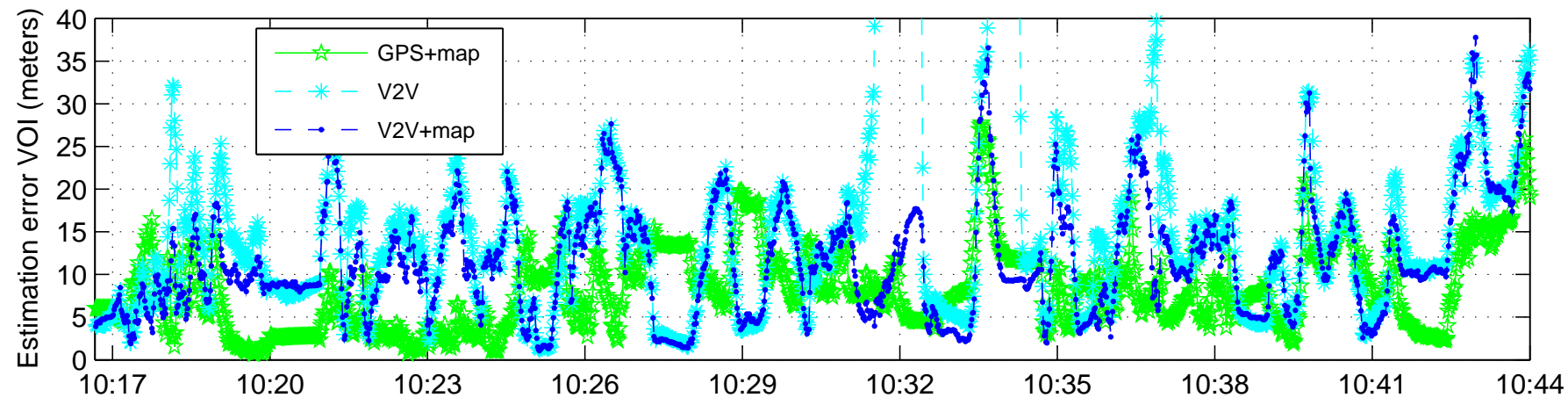

(d) Mean estimation error from 20 runs

Fig. 3: Various metrics for the whole trip displayed per second.

50 beacons per second. A GPS receiver was positioned on vehicle's rooftop, connected to the LinkBird. Inside, two Nexus 4 or 5 smartphones (near the windshield) collect inertial, Wi-Fi and GPS measurements with the maximum possible sampling rates. Video (1080p, $30 \mathrm{fps}$ ) was also captured in order to infer location ground truth. We chose the front vehicle, equipped with two cameras (front and rear) as the vehicle of interest but the results apply to all vehicles, since they act simultaneously as a vehicle of interest from their own perspective and as an anchor relative to their neighbors. The last part of the drive, in downtown area, includes some zones with poor GPS coverage due to narrow streets flanked by buildings. The estimated mean 
Data: State and weight of each particle

Result: Estimated location

get initial position and uncertainty from individual

position data or mean of anchors positions;

initialize particles' states randomly ( $3 \sigma$ area around

initial position) and weights uniformly;

foreach time instance do

get speed measurements;

get heading change from UKF;

foreach particle do

sample speed error;

sample heading error;

calculate displacement;

update state;

end

if using map restrictions then

delete particles outside road;

replicate current particles using weights;

end

if using individual position data then

get individual position and respective uncertainty;

foreach particle do

calculate Gaussian probability of location;

update weight;

end

normalize weights (sum to 1 );

end

if using $V 2 V$ ranging data then

foreach anchor do

get mean RSSI;

get individual position and uncertainty;

end

foreach particle do

foreach anchor do

if $\sigma_{a}<=15 \mathrm{~m}$ then

calculate likelihood of particle

position from RSSI, distance to the anchor and anchor uncertainty (from a previously created lookup table); update weight;

end

end

end

if sum(weight) $<$ ZeroThreshold then

restart filter;

else

| normalize weights (sum to 1 );

end

end

if $1 / \operatorname{sum}\left(\right.$ weight $\left.^{2}\right)<$ ResamplingThreshold then

delete lowest weighted particles;

copy highest weighted particles according to their

weights maintaining the total number of

particles;

normalize weights (sum to 1 );

end

get MAP estimate: the state (location and velocity) of highest weighted particle;

end

Algorithm 1: Vehicle tracking algorithm using particle filter

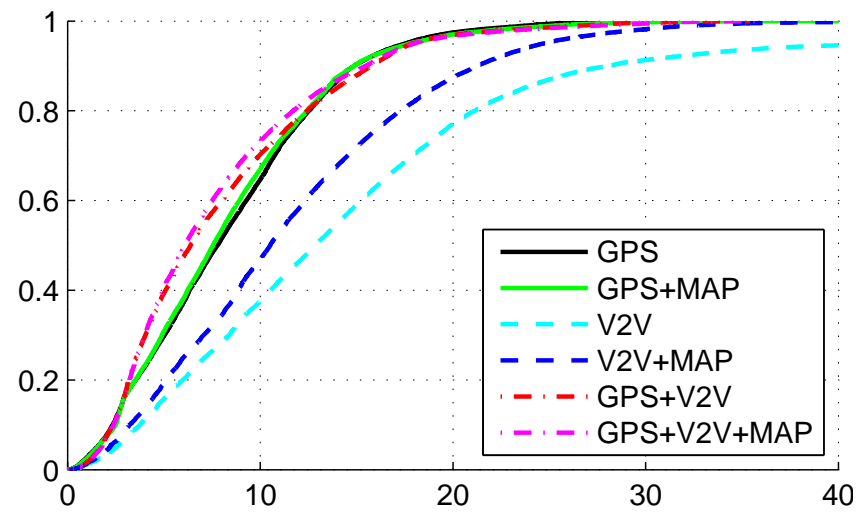

Fig. 4: CDF of location error in meters.

GPS location error of the anchors is $7 \mathrm{~m}$ for a confidence interval of $1 \sigma_{a}$. The mean GPS distance between $V_{0}$ and each of the anchors is $23.2 \mathrm{~m}, 48.3 \mathrm{~m}$, and $55.8 \mathrm{~m}$. These parameters are show in Fig. 3a and Fig. 3c, respectively, for each anchor throughout the trip. The mean RSSI values from 50 beacons per second are shown as well in Fig. 3b.

The following parameters were used in the experiments. The Resampling Threshold was set to $10 \%$ of the total number of particles and the ZeroThreshold was set to $10^{-18}$. The particle filter used 1000 particles with resampling eliminating the lowest $10 \%$ weights and replicating the top $80 \%$. It applied $T=1 \mathrm{~s}$ iterations with sub-iterations of $200 \mathrm{~Hz}$ for the motion model (UKF). The channel parameters were $\rho_{0}=-34 \mathrm{dBm}, \alpha=2.1$, and $\sigma=5.5 \mathrm{~dB}$, obtained as described in IV-A from previous experiments in similar conditions. The individual locations and respective uncertainty from the anchors were provided by their own GPS receivers from one the smartphones. If available, GPS position estimates were drawn from a Gaussian distribution with position as mean and an estimated horizontal standard deviation provided by the GPS receiver (when unavailable we used $\sigma_{\text {GPS }}=5 \mathrm{~m}$ ). The gyroscope and accelerometer from the smartphone collected data at $200 \mathrm{~Hz}$ sampling rate, and magnetometer at $50 \mathrm{~Hz}$. The speed measurement was obtained from the GPS (even when GPS is not used in the update phase) since we did not have the OBD device available. The standard deviations used in the motion model were set to $\sigma_{h}=2^{\circ} / \mathrm{s}$ and $\sigma_{s}=0.75 \mathrm{~m} / \mathrm{s}$. Map information from Open Street Map was used as described in Section IV-B. The ground truth of the position was marked manually using videos as the main source. Two different videos were obtained from cameras at the front and back of the vehicle of interest, as well as at the front of all the vehicles following it. In addition, we used the map and GPS from various devices to disambiguate some situations (when the videos did not provide clear landmarks). We choose the smartphone attached to the mid-section of the windshield (containing the front camera and collecting GPS data) as the true position of the vehicle. Although the trajectory may be very close to the real one, some error introduced by the manual labeling is unfortunately inevitable at high speeds.

The results for 20 runs of the algorithm are presented in Table I for six different combinations of the three location 
TABLE I: Location errors for full trajectory (meters)

\begin{tabular}{|l|c|c|c|c|c|c|}
\hline $\begin{array}{l}\text { Location } \\
\text { sources }\end{array}$ & GPS & $\begin{array}{c}\text { GPS } \\
+ \text { map }\end{array}$ & V2V & $\begin{array}{c}\text { V2V } \\
+ \text { map }\end{array}$ & $\begin{array}{c}\text { GPS } \\
+ \text { V2V }\end{array}$ & $\begin{array}{c}\text { GPS } \\
+ \text { V2V } \\
+ \text { map }\end{array}$ \\
\hline RMSE & 9.80 & 9.84 & 25.71 & 13.63 & 9.68 & 9.47 \\
MAE & 8.37 & 8.31 & 17.01 & 11.60 & 7.92 & 7.70 \\
SD [heading] & 2.68 & 2.51 & 4.70 & 3.03 & 2.26 & 2.10 \\
SD [ $\perp$ heading] & 2.90 & 2.62 & 5.51 & 3.25 & 2.39 & 2.19 \\
\hline
\end{tabular}

information sources: GPS, RSSI ranging information denominated $\mathrm{V} 2 \mathrm{~V}$ for simplicity, and map restrictions. We show the root-mean-square error (RMSE), the mean absolute error (MAE), which is the mean of location error, and the standard deviation (SD) of the posterior function, providing a measure of confidence in the estimated position, both in the direction of motion (heading), that will mostly coincide with the direction of the road, and in the perpendicular direction. In order to have a better picture of the errors we also present the cumulative distribution function (CDF) of the location error in meters in Fig. 4. We observe that both the maps and the $\mathrm{V} 2 \mathrm{~V}$, in combination with GPS, improve its results and, as we would expect, all three sources of information together provide the best configuration. The position accuracy achieved in this case is less than $6.1 \mathrm{~m}$ for $50 \%$ of the trip, and less than $11.7 \mathrm{~m}$ for $80 \%$. It is important to note that not only the accuracy is better, but also the reliability of the results is improved in comparison to using only a subset of the available information sources. The V2V based model, with no GPS available in the vehicle of interest or fixed anchors with known exact positions, relying on the GPS estimates of its neighbors, often out-of-reach or in poorly covered GPS areas, is able to provide localization with a position accuracy of $12.8 \mathrm{~m}$ for $50 \%$ of the trip, even without the map. It's relevant to point out that this configuration shows a small percentage of very large errors $(5 \%$ of the errors are higher than $40 \mathrm{~m}$ ) from a specific situation where $V_{0}$ was separated from the rest of the vehicles, which got delayed by a traffic light (see in Fig. 3 the interval of 1 minute centered in 10:32), resulting not only in very large distances between the vehicle of interest and the anchors but also in a especially poor anchor geometry. In fact, very close to each other from a large distance of $V_{0}$, these 3 anchors behave as virtually only one and the likelihood takes an annular shape, not allowing the model to distinguish the correct road in a bifurcation. When combining map restrictions, we are able avoid these high errors and improve significantly the quality of the overall estimation. The error for the $\mathrm{V} 2 \mathrm{~V}$ configuration combined with map restrictions is less than $10.5 \mathrm{~m}$ in $50 \%$ of the cases and less than $17.3 \mathrm{~m}$ in $80 \%$. We observe that the use of map restrictions, particularly when combined with $\mathrm{V} 2 \mathrm{~V}$, improves both the accuracy and the reliability of the estimation significantly.

In Fig. 3d, we show the values of the location error (MAE) throughout the trip, allowing the observation of its relation with the error of the anchors and the distance to $V_{0}$, for the configurations without GPS, and provide the GPS with maps as a benchmark. We confirm that the moments for which the V2V errors are larger, coincide with the situations where all three anchors were at large distances from the vehicle of
TABLE II: Location errors for intervals with good conditions of the anchors (meters)

\begin{tabular}{|l|c|c|c|c|c|c|}
\hline $\begin{array}{l}\text { Location } \\
\text { sources }\end{array}$ & GPS & $\begin{array}{c}\text { GPS } \\
+ \text { map }\end{array}$ & V2V & $\begin{array}{c}\text { V2V } \\
+ \text { map }\end{array}$ & $\begin{array}{c}\text { GPS } \\
+ \text { V2V }\end{array}$ & $\begin{array}{c}\text { GPS } \\
+ \text { V2V } \\
+ \text { map }\end{array}$ \\
\hline RMSE & 9.62 & 9.23 & 9.60 & 8.70 & 7.30 & 6.93 \\
MAE & 8.64 & 8.28 & 8.05 & 7.32 & 6.18 & 5.90 \\
SD [heading] & 2.08 & 2.10 & 2.57 & 2.18 & 1.58 & 1.54 \\
SD [ $\perp$ heading] & 2.25 & 2.03 & 2.66 & 2.28 & 1.68 & 1.58 \\
\hline
\end{tabular}

interest. It is very important to stress that in these experiments we were limited to those three vehicles as anchors. They were often distant (sometimes even completely out of reach) and providing a poor anchor geometry. However, in a typical scenario of a densely populated urban area, a larger number of vehicles is expected to be within communication reach of $V_{0}$ and the ones providing the best geometry and lowest position errors might be chosen as anchors. We present in Table II, the results only for moments that present favorable conditions to apply $\mathrm{V} 2 \mathrm{~V}$, namely all three anchors close to the leading vehicle (less than 40m of GPS distance) and with good position accuracy (less than $8 \mathrm{~m}$ for $1 \sigma_{a}$ confidence interval). In our experiment these happened approximately $23 \%$ of the trip, but we expect them to occur much more frequently in large vehicular networks. We observe that for these favorable conditions, the performance of our tracking algorithm even when $V_{0}$ has no access to GPS position is quite good. The location error of the $\mathrm{V} 2 \mathrm{~V}$ based configuration is $8 \mathrm{~m}$ and the estimation has a standard deviation of approximately $2.6 \mathrm{~m}$, which is a performance equivalent to the GPS for the same context. The accuracy of the configuration $\mathrm{V} 2 \mathrm{~V}+$ map is $7.3 \mathrm{~m}$, exceeding the accuracy of the GPS+map, which is $8.3 \mathrm{~m}$. This shows the potential of our proposed model. It is also interesting to note that, since the anchors follow the leading vehicle, the distribution of the vehicles in space is mostly in line or in two lanes in the same direction, which is a poor geometry (see Fig. 5). However, while this limits more the longitudinal error (along the direction of the $\mathrm{road} /$ movement, using map restrictions bounds the lateral error (perpendicular to the previous direction), leading to a balanced combination of information sources. In our implementation the road restrictions assume a worse case road width, so the impact is mostly noticeable in situations for which the errors are very high (e.g. very distant anchors). Nevertheless, using more tight road constraints, for example having access to the actual width of each road, would lead to an even higher impact on location performance.

\section{CONCLUSION}

We propose a location tracking approach for vehicular networks, allowing vehicles to estimate or improve their position using widely available low-cost smartphone sensors and information shared by one-hop neighbors with uncertain locations. We provide a proof-of-concept using data from a real urban scenario with four communicating vehicles, collecting their GPS, RSSI, and inertial data, and using available road maps. We present results evaluating the quality of different combinations of these information sources. By combining all 


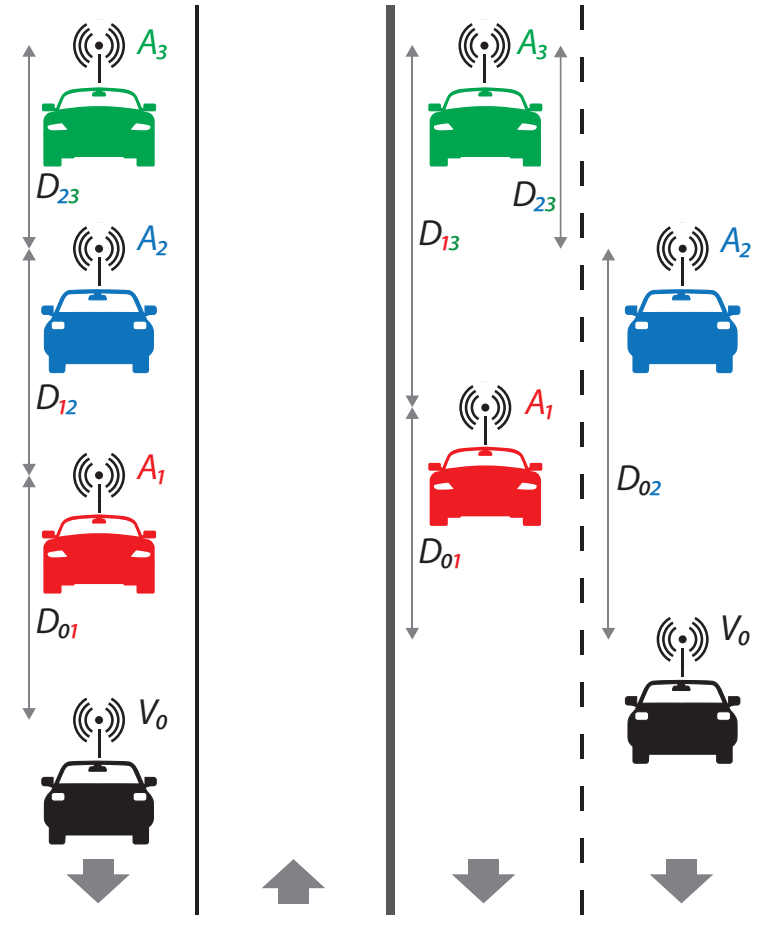

Fig. 5: Geometry of anchors and $V_{0}$ during the trip: longitudinal distances (measured along the road) $D_{i j} \geq 0$ between the vehicles' antennas vary in time and represent the distribution of vehicles in space. The leading vehicle is $V_{0}$ and $A_{1}, A_{2}$ and $A_{3}$ follow in that order (their colors correspond to the ones used to represent them in Fig. 3), either in one lane or in two lanes when possible. Except for very close distances, both geometries have a similar impact. For large distances the configuration on the right can be approximated by the one on the left, so the geometry is mostly in line following the VOI.

of them, we provide a mean location error of $7.7 \mathrm{~m}$ during the whole trip, including urban downtown areas with low GPS coverage, as well as relying on anchors with uncertain and often poor position estimates and geometry. For intervals with good conditions of the anchors, we reduce this value to $5.9 \mathrm{~m}$. It is relevant to highlight that by leveraging different sources of information not only the accuracy is improved, but the model is also more reliable and robust to failure. We propose the existence of at least 3 neighbor vehicles with good position accuracy (less than $8 \mathrm{~m}$ for $1 \sigma_{a}$ confidence interval) and a maximum distance of $40 \mathrm{~m}$ to the VOI as favorable conditions for using $\mathrm{V} 2 \mathrm{~V}$ ranging. In these $\mathrm{V} 2 \mathrm{~V}$ conditions, we show it is possible to provide a performance comparable to GPS even when the VOI lacks access to GPS, achieving a mean location error of approximately $8 \mathrm{~m}$. These results motivate us to explore more cooperative scenarios, and extend our model to be applied in a real setting by taking advantage of the currently largest urban vehicular network in the world, deployed in the city of Porto, Portugal [41].

\section{ACKNOWLEDGMENT}

The authors would like to thank their colleagues Rui Meireles, Pedro Santos, and João Rodrigues from IT Porto for their help with the data collection experiments.

\section{REFERENCES}

[1] A. Boukerche, H. Oliveira, E. Nakamura, and A. Loureiro, "Vehicular ad hoc networks: A new challenge for localization-based systems," Computer communications, vol. 31, no. 12, pp. 2838-2849, 2008.

[2] I. Skog and P. Händel, "In-car positioning and navigation technologies - A survey," IEEE Transactions on Intelligent Transportation Systems, vol. 10, no. 1, pp. 4-21, 2009.

[3] H. Wymeersch, J. Lien, and M. Z. Win, "Cooperative localization in wireless networks," Proceedings of the IEEE, vol. 97, no. 2, pp. 427450, 2009.

[4] P. Papadimitratos, A. de La Fortelle, K. Evenssen, R. Brignolo, and S. Cosenza, "Vehicular communication systems: Enabling technologies, applications, and future outlook on intelligent transportation," IEEE Communications Magazine, vol. 47, no. 11, pp. 84-95, 2009.

[5] N. Patwari, J. N. Ash, S. Kyperountas, A. O. Hero III, R. L. Moses, and N. S. Correal, "Locating the nodes: cooperative localization in wireless sensor networks," IEEE Signal Processing Magazine, vol. 22, no. 4, pp. $54-69,2005$.

[6] T. E. Abrudan, L. M. Paula, J. Barros, J. P. S. Cunha, and N. B. Carvalho, "Indoor location estimation and tracking in wireless sensor networks using a dual frequency approach," in 2011 IEEE International Conference on Indoor Positioning and Indoor Navigation (IPIN), Guimarães, Portugal, 21-23 Sep. 2011.

[7] T. E. Abrudan, A. Haghparast, and V. Koivunen, "Time-synchronization and ranging for OFDM systems using time-reversal," IEEE Transactions on Instrumentation and Measurement, vol. 62, no. 12, pp. 3276-3290, 2013.

[8] S. Gezici, Z. Tian, G. B. Giannakis, H. Kobayashi, A. F. Molisch, H. V. Poor, and Z. Sahinoglu, "Localization via ultra-wideband radios: a look at positioning aspects for future sensor networks," IEEE Signal Processing Magazine, vol. 22, no. 4, pp. 70-84, July 2005.

[9] S. Bartoletti, W. Dai, A. Conti, and M. Z. Win, "A mathematical model for wideband ranging," Selected Topics in Signal Processing, IEEE Journal of, vol. 9, no. 2, pp. 216-228, 2015.

[10] S. Bartoletti, A. Giorgetti, M. Z. Win, and A. Conti, "Blind selection of representative observations for sensor radar networks," Vehicular Technology, IEEE Transactions on, vol. 64, no. 4, pp. 1388-1400, 2015.

[11] L. Oliveira, C. Di Franco, T. E. Abrudan, and L. Almeida, "Fusing time-of-flight and received signal strength for adaptive radio-frequency ranging," in 16th Conference on Advanced Robotics (ICAR 2013), Montevideo, Uruguay, 25-29 Nov. 2013.

[12] T. E. Abrudan, Z. Xiao, A. Markham, and N. Trigoni, "Distortion rejecting magneto-inductive three-dimensional localization (MagLoc)," IEEE Journal on Selected Areas in Communications, vol. 33, no. 11, pp. 2404-2417, Nov. 2015.

[13] M. Youssef and A. Agrawala, "The Horus WLAN location determination system," in MobiSys, 2005.

[14] S. Thrun, W. Burgard, and D. Fox, Probabilistic Robotics. Cambridge: The MIT Press, 2005.

[15] E. I. Laftchiev, C. M. Lagoa, and S. N. Brennan, "Vehicle localization using in-vehicle pitch data and dynamical models," IEEE Transactions on Intelligent Transportation Systems, vol. 16, no. 1, pp. 206-220, 2015.

[16] S. Rezaei and R. Sengupta, "Kalman filter-based integration of DGPS and vehicle sensors for localization," IEEE Transactions on Control Systems Technology, vol. 15, no. 6, pp. 1080-1088, 2007.

[17] M. A. Quddus, W. Y. Ochieng, and R. B. Noland, "Current mapmatching algorithms for transport applications: State-of-the art and future research directions," Transportation Research Part C: Emerging Technologies, vol. 15, no. 5, pp. 312-328, 2007.

[18] E. Parliament and Council, "Directive 2010/40/EU," 7 July 2010, URL: http://eur-lex.europa.eu/eli/dir/2010/40/oj.

[19] M. Z. Win, A. Conti, S. Mazuelas, Y. Shen, W. M. Gifford, D. Dardari, and M. Chiani, "Network localization and navigation via cooperation," Communications Magazine, IEEE, vol. 49, no. 5, pp. 56-62, 2011.

[20] Y. Shen, S. Mazuelas, and M. Z. Win, "Network navigation: Theory and interpretation," Selected Areas in Communications, IEEE Journal on, vol. 30, no. 9, pp. 1823-1834, 2012.

[21] M. Efatmaneshnik, N. Alam, A. T. Balaei, A. Kealy, and A. G. Dempster, "Cooperative positioning in vehicular networks," Wireless Technologies in Vehicular Ad Hoc Networks: Present and Future Challenges. IGI Global, Mexico City, ISBN, vol. 1826069098, pp. 245-270, 2012.

[22] D. Fox, W. Burgard, H. Kruppa, and S. Thrun, "A probabilistic approach to collaborative multi-robot localization," Autonomous robots, vol. 8, no. 3, pp. 325-344, 2000. 
[23] J. J. Blum, A. Eskandarian, and L. J. Hoffman, "Challenges of intervehicle ad hoc networks," IEEE Transactions on Intelligent Transportation Systems, vol. 5, no. 4, pp. 347-351, 2004.

[24] R. Parker and S. Valaee, "Vehicular node localization using receivedsignal-strength indicator," IEEE Transactions on Vehicular Technology, vol. 56, no. 6, pp. 3371-3380, 2007.

[25] N. M. Drawil and O. Basir, "Intervehicle-communication-assisted localization," IEEE Transactions on Intelligent Transportation Systems, vol. 11, no. 3, pp. 678-691, 2010.

[26] N. Mattern, M. Obst, R. Schubert, and G. Wanielik, "Co-operative vehicle localization algorithm-Evaluation of the CoVeL approach," in 9th International Multi-Conference on Systems, Signals and Devices (SSD). IEEE, 2012, pp. 1-5.

[27] M. Obst, N. Mattern, R. Schubert, and G. Wanielik, "Car-to-car communication for accurate vehicle localization-the CoVeL approach," in 9th International Multi-Conference on Systems, Signals and Devices (SSD). IEEE, 2012, pp. 1-6.

[28] K. Liu and H. B. Lim, "Positioning accuracy improvement via distributed location estimate in cooperative vehicular networks," in 15th International IEEE Conference on Intelligent Transportation Systems (ITSC). IEEE, 2012, pp. 1549-1554.

[29] N. Alam, A. Tabatabaei Balaei, and A. G. Dempster, "Relative positioning enhancement in VANETs: A tight integration approach," IEEE Transactions on Intelligent Transportation Systems, vol. 14, no. 1, pp. 47-55, 2013.

[30] H. Li and F. Nashashibi, "Multi-vehicle cooperative localization using indirect vehicle-to-vehicle relative pose estimation," in ICVES 2012IEEE International Conference on Vehicular Electronics and Safety, 2012 , pp. $267-272$.

[31] — "Cooperative multi-vehicle localization using split covariance intersection filter," Intelligent Transportation Systems Magazine, IEEE, vol. 5, no. 2, pp. 33-44, 2013.

[32] G. Mao, B. Fidan, and B. Anderson, "Wireless sensor network localization techniques," Computer networks, vol. 51, no. 10, pp. 2529-2553, 2007.

[33] M. Angjelichinoski, D. Denkovski, V. Atanasovski, and L. Gavrilovska, "Cramér-Rao lower bounds of RSS-based localization with anchor position uncertainty," Information Theory, IEEE Transactions on, vol. 61, no. 5, pp. 2807-2834, 2015.

[34] Y. Shen and M. Z. Win, "Fundamental limits of wideband localizationpart i: A general framework," Information Theory, IEEE Transactions on, vol. 56, no. 10, pp. 4956-4980, 2010.

[35] J. V. Candy, Bayesian Signal Processing: Classical, Modern and Particle Filtering Methods. John Wiley \& Sons, 2009.

[36] A. Conti, D. Dardari, M. Guerra, L. Mucchi, and M. Z. Win, "Experimental characterization of diversity navigation," Systems Journal, IEEE, vol. 8, no. 1, pp. 115-124, 2014

[37] S. J. Julier and J. K. Uhlmann, "New extension of the Kalman filter to nonlinear systems," in AeroSense'97. International Society for Optics and Photonics, 1997, pp. 182-193.

[38] F. Daum and J. Huang, "Curse of dimensionality and particle filters," vol. 4, pp. 1979-1993, 2003.

[39] F. Daum, "Nonlinear filters: beyond the Kalman filter," IEEE Aerospace and Electronic Systems Magazine, vol. 20, no. 8, pp. 57-69, 2005.

[40] T. Harada, T. Mori, and T. Sato, "Development of a tiny orientation estimation device to operate under motion and magnetic disturbance," The International Journal of Robotics Research, vol. 26, no. 6, pp. 547$559,2007$.

[41] Future Cities Project, "Discussing the largest vehicular network in the world," April 2015. [Online]. Available: http://futurecities.up.pt/site/discussing-the-largest-vehicularnetwork-in-the-world/

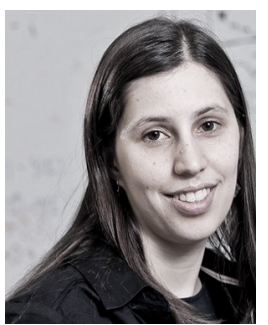

Susana B. Cruz received her M.Sc. degree in Electrical and Computer Engineering from the Faculty of Engineering University of Porto (FEUP), Portugal, in 2010, and is currently pursuing the Ph.D. degree in the same institution. She develops her work at Instituto de Telecomunicações (IT) Porto and was awarded a Doctoral Scholarship from the Portuguese Foundation for Science and Technology (FCT) in 2010. Her research interests include wireless sensor networks and intelligent transportation systems, focusing on vehicular networks, localization and

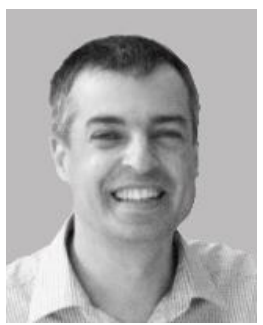

Traian E. Abrudan (S'02, M'09) received the D.Sc. degree (with honors) from Aalto University, Finland (formerly known as Helsinki University of Technology) in 2008, and the M.Sc. degree from the Technical University of Cluj-Napoca, Romania in 2000. During 2010-2013, he was a Postdoctoral Researcher with the Faculty of Engineering, University of Porto (FEUP), Portugal, and member of Instituto de Telecomunicações (IT) Porto. During 2013-2016, he was a Postdoctoral Researcher with the Department of Computer Science, University of Oxford, Oxford, UK. In September 2016, he joined Nokia Bell Labs. His fundamental research topics include sensor array signal processing, applied parameter estimation, numerical optimization, and wireless transceiver algorithms.

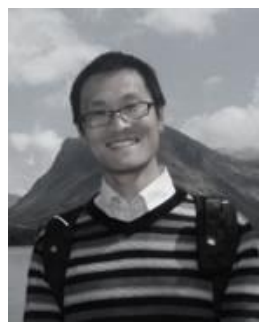

Zhuoling Xiao (S'14, M'16) received the Ph.D. degree from the University of Oxford, Oxford, U.K., in 2015.

His research interests focus on sensor networks, including localization, communication, and coordination protocols for networked sensor nodes and machine learning techniques for sensor networks and localization.

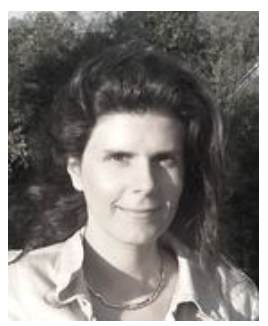

Niki Trigoni is a Professor at the Department of Computer Science, University of Oxford. She obtained her $\mathrm{PhD}$ at the University of Cambridge (2001), became a postdoctoral researcher at Cornell University (2002-2004), and a Lecturer at Birkbeck College (2004-2007). Since she moved to Oxford in 2007, she established the Sensor Networks Group, and has conducted research in communication, localization and in-network processing algorithms for sensor networks. Her recent and ongoing projects span a wide variety of sensor networks applications, including indoor/underground localization, wildlife sensing, road traffic monitoring, autonomous (aerial and ground) vehicles, and sensor networks for industrial processes.

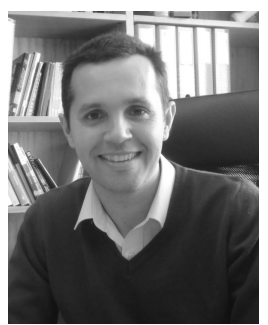

João Barros (S'98, M'04, SM'11) is an Associate Professor of Electrical and Computer Engineering at the University of Porto and Founding Director of the Institute for Telecommunications (IT) in Porto, Portugal. He also teaches at the Porto Business School and co-founded two recent startups, Streambolico and Veniam, commercializing wireless video and vehicular communication technologies, respectively. He received his undergraduate education in Electrical and Computer Engineering from the Universidade do Porto (UP), Portugal and Universitaet Karlsruhe, Germany, and the Ph.D. degree in Electrical Engineering and Information Technology from the Technische Universitaet Muenchen (TUM), Germany. 